\title{
GLENOUMERAL DISLOCATION: A PROSPECTIVE RANDOMIZED STUDY COMPARING SPAZO AND KOCHER MANEUVERS
}

\author{
Bruno da Rocha Moreira Rezende ${ }^{1}$, José Inácio de Almeida Neto ${ }^{1}$, Uriel Jaime de Sousa ${ }^{1}$, Leônidas de Souza Bomfim ${ }^{1}$, \\ MÁrIo SOARES FERREIRA JÚNIOR ${ }^{1}$
}

\section{ABSTRACT}

Objective: To evaluate and to compare the Spaso and Kocher reduction maneuvers in terms of efficiency, time of reduction, facileness, pain, discomfort to the patient, complications and risks, besides promoting the comparison between the two maneuvers, aiming to ground the emergency conduct. Methods: A prospective study with 105 patients with acute shoulder dislocations were enrolled in the study between February 2011 and September 2012. The patients were randomized into two groups and they were submitted to the reduction of dislocation using the Spaso maneuver (group A) or the Kocher technique (group B) by first, second or third-year orthopedic surgery residents from our service. The results were evaluated and compared. Results: There was no statistically significant difference between the two groups in terms of age, gender, timing of reduction, number of precedent episodes and complications after reduction. However, reduction was achieved in more cases using the Spaso method than it was among the Kocher group, as well as the mean duration of the reduction maneuver and discomfort were shorter in the group A patients. Conclusions: Both methods presented good results in terms of dislocation reduction and low complications rates. Nevertheless, the Spaso maneuver was more efficient, fast and easily applicable in comparison with Kocher's method. Level of Evidence I, Therapeutic.

Keywords: Shoulder dislocations. Shoulder joint. Manipulation, orthopedic/methods. Prospective studies.

Citation: Rezende BRM, Almeida Neto Jl, Sousa UJ, Bomfim LS, Ferreira Júnior MS. Glenoumeral dislocation: a prospective randomized study comparing Spazo and Kocher maneuvers. Acta Ortop Bras. [online]. 2015;23(4):192-6. Available from URL: http://www.scielo.br/aob.

\section{INTRODUCTION}

The glenohumeral dislocation is an extremely common event in orthopedic emergencies, corresponding to approximately $50 \%$ of all dislocations, and the knowledge of their management and treatment is the duty of every orthopedist.

It is known that there are numerous reduction techniques described, ranging from Hippocrates ${ }^{1}$, through Milch, ${ }^{2}$ Stimson, Kocher, ${ }^{3,4}$ traction and counter-traction, beyond the Spaso technique published by Miljesic and Kelly ${ }^{4}$, still little known in our midst, despite some good results in the few preliminary studies in the literature. ${ }^{5}$

All these approach options present, in turn, the most varied success and complication rates, such as: non reduction, iatrogenic fracture, neurovascular injury, soft tissue injury, medical team distress, pain and patient discomfort.

Despite the high incidence of shoulder dislocation and its importance in the orthopedic field, there are not, however, studies in the literature that compare the most diverse techniques regarding those mentioned aspects. Its indication is usually associated with experience and personal choice, unfounded on scientific evidence (most of the recommendations levels 4 and 5).

The objective of this study was to evaluate a traditional technique known by most orthopedists - Kocher's technique - to a most recent one, the Spaso maneuver, which does not has yet many references in the literature; however, it has shown excellent results in a few isolated published studies. We also seek to compare both techniques regarding efficacy, reduction time, applicability, patient comfort and complications.

\section{MATERIALS AND METHODS}

This study was a prospective, randomized clinical trial, approved by our institutional Ethics Research Committee (ERC), and it meets the standards of the National Research Ethics Com-

All the authors declare that there is no potential conflict of interest referring to this article.

1. Hospital de Base do Distrito Federal, Brasilia, DF, Brazil.

Work developed at Hospital de Base do Distrito Federal, Department of Orthopedics, Brasilia, DF, Brazil.

Correspondence: SQN 203 Bloco K, apartamento 501, Asa Norte, Brasília, DF, Brazil. brunorezende@arthrosortopedia.com 
mission (Comissão Nacional de Ética em Pesquisa, CONEP), being duly registered by the number 05998412.0.0000.5553. All patients included in the study signed the Free and Informed Consent form, having been previously informed on the nature of the study and their participation in it.

Between February 2011 and September 2012, 105 patients with traumatic anterior glenohumeral dislocation were seen in the Traumatology and Orthopedics Emergency Service in our hospital. After performing the appropriate propaedeutic - complete physical examination (including neurovascular evaluation) and shoulder radiographs on trauma series - all patients with confirmed diagnosis of dislocation were included in the study, since they did not present any exclusion criteria: use of analgesics after dislocation or fracture of the proximal humerus, unconsciousness, refusal to sign the consent form, failure to fill the evaluation form.

Patients were therefore randomized into two groups arbitrarily by means of previously numbered charts, being pair chart number corresponding to group $A$ and even chart number to group B (zero was considered as even). The first group was submitted to reduction by the Spaso maneuver and the second using Kocher technique. Reductions were performed by a single Orthopedics and Traumatology resident physician, $\left(1^{\text {st }}, 2^{\text {nd }}\right.$ or $3^{\text {rd }}$ year), and the time required for reduction was timed with a standard clock by an assistant who did not participate in the maneuver. The resident had three minutes to perform the reduction through the chosen maneuver, which occurred without previous administration of analgesics, anesthetics or muscle relaxants; after this period, in case of failure, he was free to try other methods. An evaluation form was filled, which contained the name, registration, age, gender, affected side, dislocation time, number of previous events, fracture or any neurovascular alteration detected before the procedure, no reduction of the dislocation, and the time of reduction, resident year, comments, complications, and subjective evaluation of pain reported by the patients using a visual analogue pain scale (VAS). All residents had previous training - theoretical and practical - regarding the two maneuvers used.

\section{Statistical analysis}

Determining the sample size required to reject the null hypothesis with $\alpha$ value set at 0.05 was obtained from the literature review, drawing upon the effectiveness of the two reduction methods reported in several published and evaluated studies. Obtaining the significant difference in efficiency ratios described for the two maneuvers of approximately $11 \%$, with the power of the test for the proposed hypothesis set at $80 \%$, and the bicaudal test significance level at $5 \%$, the Lehr ${ }^{6}$ simplified formula was used to estimate the number of patients in each group, which was 43 patients.

The descriptive analysis was performed using standard methods, Student t-test for demographic variables with significance level $\alpha$ of 0.05 (all bicaudal tests), the Chi-square test was used to compare the effectiveness between the two methods and the Wilcoxon-Mann-Whitney test was used to compare pain and time needed for reduction the between groups $\mathrm{A}$ and $\mathrm{B} .{ }^{7}$ The Spaso technique, ${ }^{4}$ according to Miljesic and Kelly, ${ }^{4}$ must be performed with the patient in supine position, with his shoulder flexed to $90^{\circ}$, elbow extended and the doctor keeping longitu- dinal traction in the affected limb to promote the elevation of the ipsilateral scapula from the exam table. (Figure 1) Traction is maintained until the patient can relax enough to allow his scapula to touch the stretcher. The shoulder, right now, should be externally gently rotated to promote the reduction. (Figure 2) Finally, the internal rotation and extension of the already reduced limb is performed. (Figure 3)

The Kocher maneuver ${ }^{1,8}$ is described with the patient in the supine position, the affected limb adducted along the body and the elbow flexed at $90^{\circ}$. (Figure 4) The shoulder is then submitted to external rotation until resistance. (Figure 5) Subsequently, the patient's arm is gently flexed maintaining the external rotation. (Figure 6) Then, limb adduction takes place. Finally, we proceed to internal rotation and extension, with the reduction of the shoulder. (Figure 7)

\section{RESULTS}

A total of 105 patients were seen by the residents of the service, during the study period, 17 having been excluded from the study: two were unconscious, six had concomitant fracture, five were on previous medication (analgesic, sedative or muscle relaxant) and in four cases not enough data was obtained. Thus, 88 patients were included in the study, 75 men (85.23\%) and 13 women (14.77\%), with no statistical difference between the groups. The mean age was $30.92 \pm 12.32$ years old; being $30.356 \pm 9.604$ years in group $A$ and $30.954 \pm 10.961$ years in group $B$, with no statistical difference between them. The right side was affected in 55 cases (62.5\%), 28 in group A (62.2\%) and 27 in group B (62.8\%). Both groups were also similar regarding the time elapsed between the dislocation and the reduction, which averaged $1.316 \pm 2.067 \mathrm{~h}$ in group $\mathrm{A}$ and $2.081 \pm 1.645 \mathrm{~h}$ in group $\mathrm{B}$. And yet, regarding the number of previous episodes, it was $3.156 \pm 3.49$ in group $A$ and $1.907 \pm 2.234$ in $\mathrm{B}$.

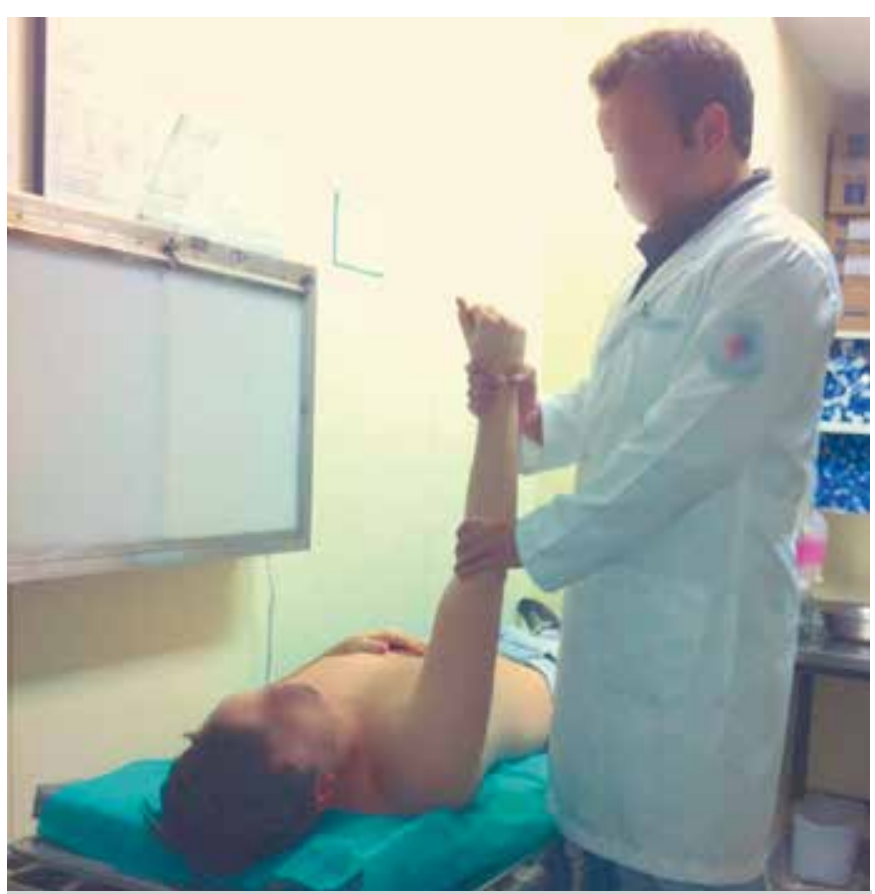

Figure 1. Longitudinal traction 


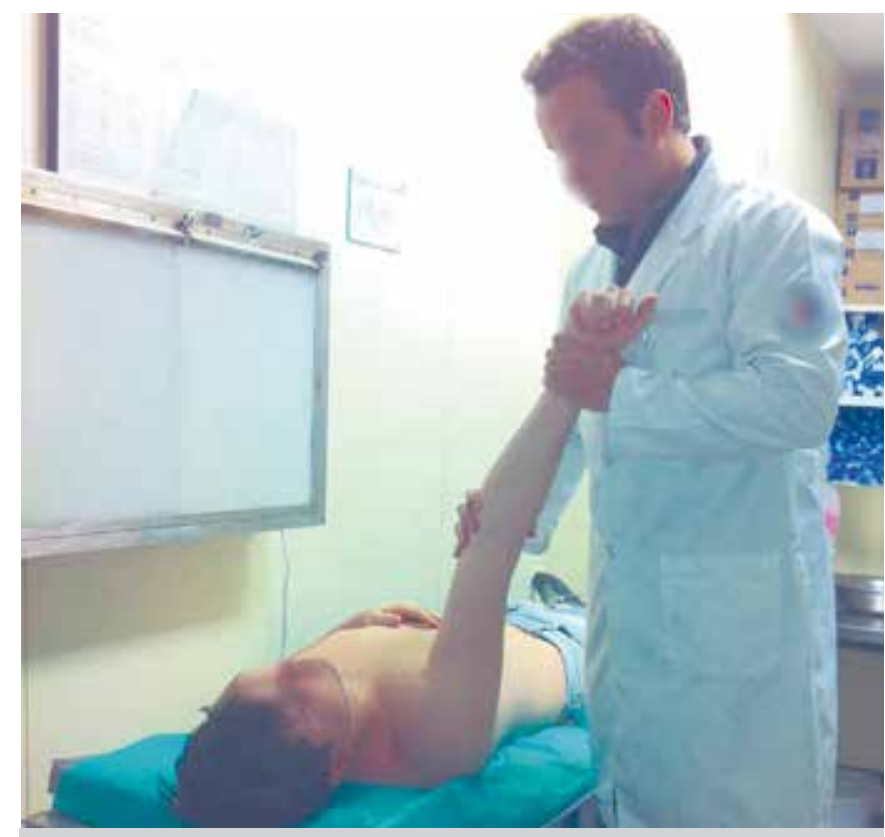

Figure 2. External rotation.

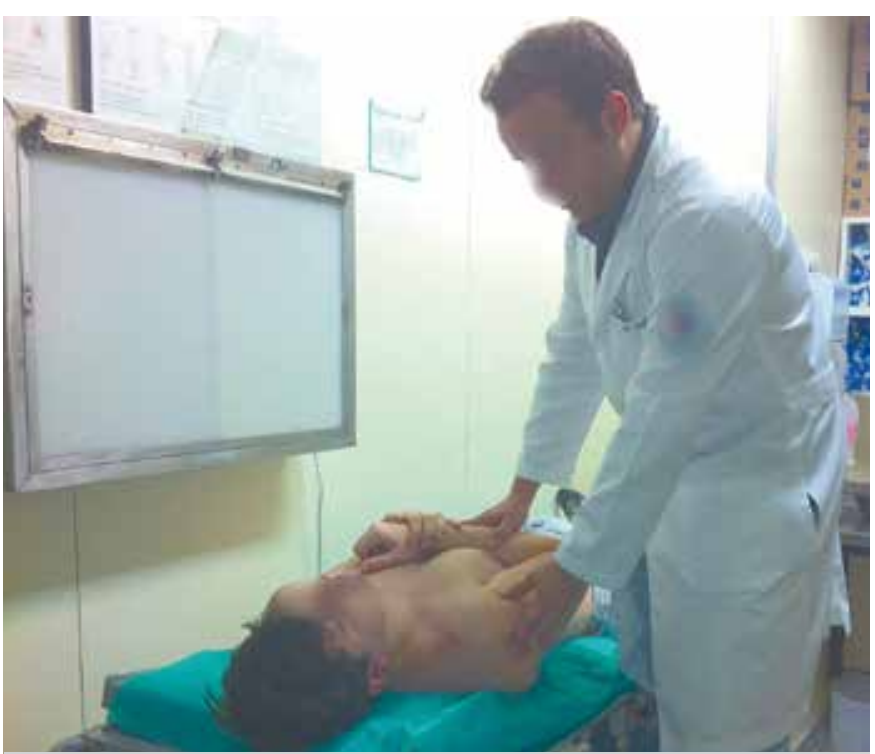

Figure 3. Internal rotation and extension.

There was thus no statistically significant difference between groups regarding to demographic variables: age, gender, affected side, time of dislocation and previous episodes. There was no complication due to the reduction in any of them. (Table 1)

Reduction was achieved in 40 patients of the Spaso group (88.9\%) and 30 patients of the Kocher group (69.77\%), making a statistically significant difference between them $(p=0.035)$. The remaining patients had their shoulders reduced by another method (4 in group A and 10 in Group B); or under anesthesia in the operating room (1 in Group A and 3 in group B).

The time required for the reduction was significantly lower in group A, $46.75 \mathrm{sec}$ on average (range 5-180 sec) when compared to Group B, $213.837 \mathrm{sec}$ on average (range 30-180 sec),

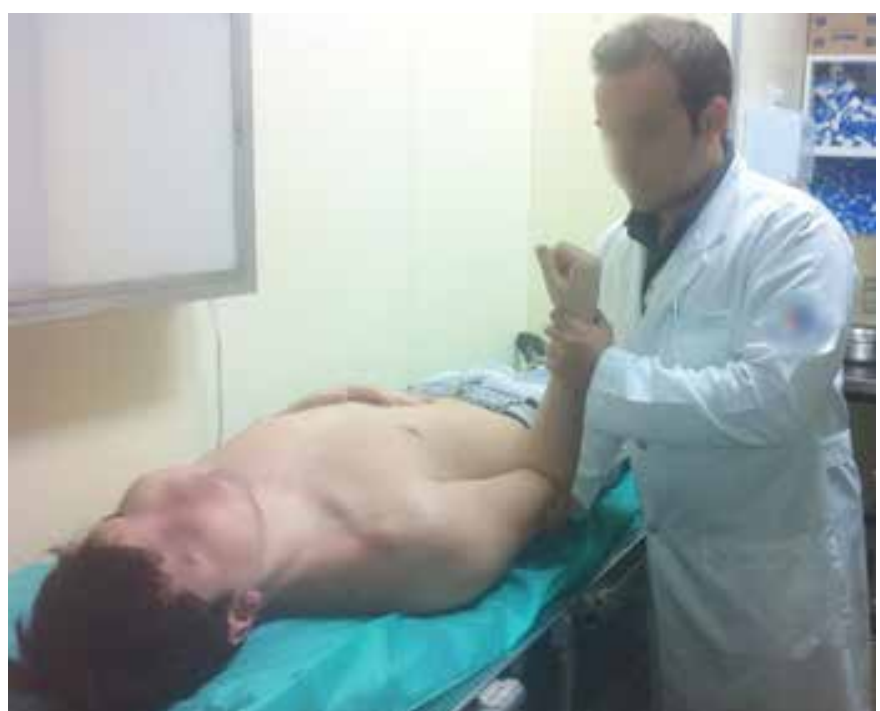

Figure 4. Elbow in adduction is flexed at $90^{\circ}$.

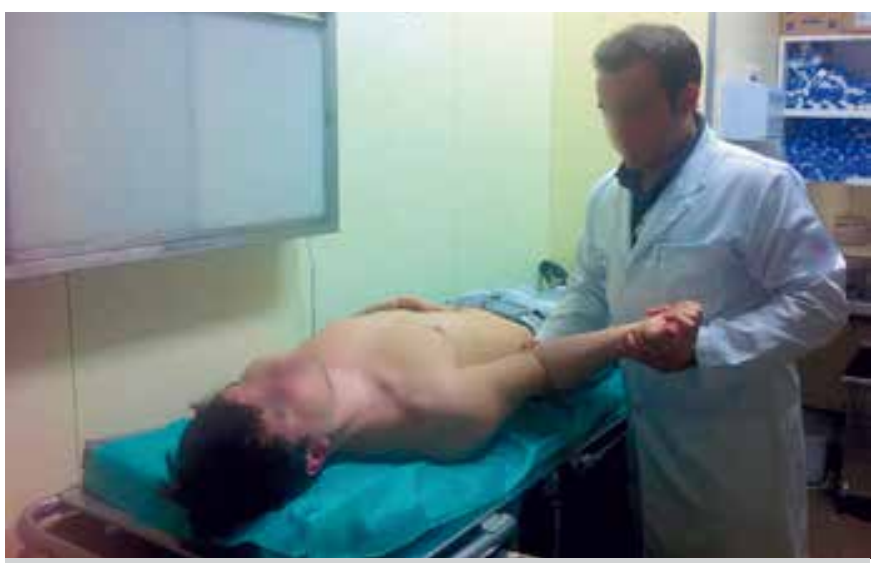

Figure 5. External rotation until resistance.

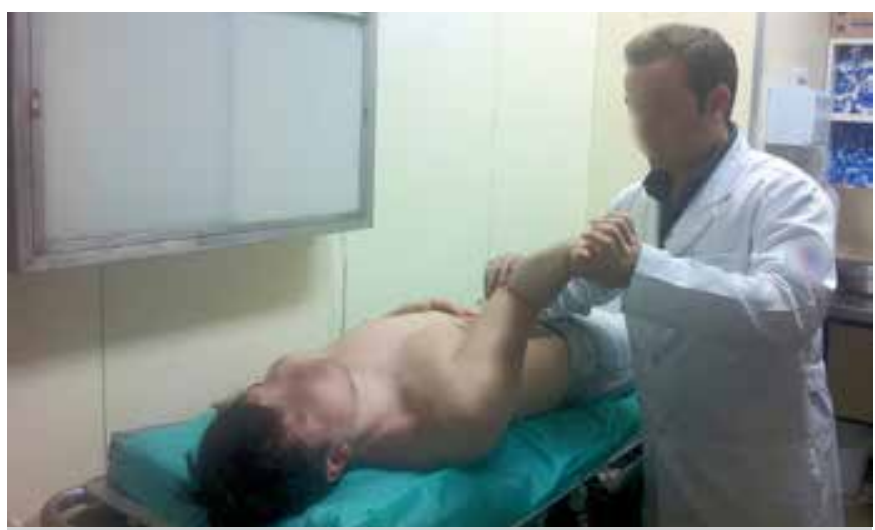

Figure 6. Shoulder adduction in external rotation.

with a p-value calculated by the Mann-Whitney-Wilcoxon method lower than 0.005 , as well as the discomfort reported by the patient, measured by the Visual Analogue Scale which was of 3.133 (1-5 of variance) on average in the Spaso group and 3.302 (range 2-5) in the Kocher group, reflecting a p value of 0.433. (Table 2). 


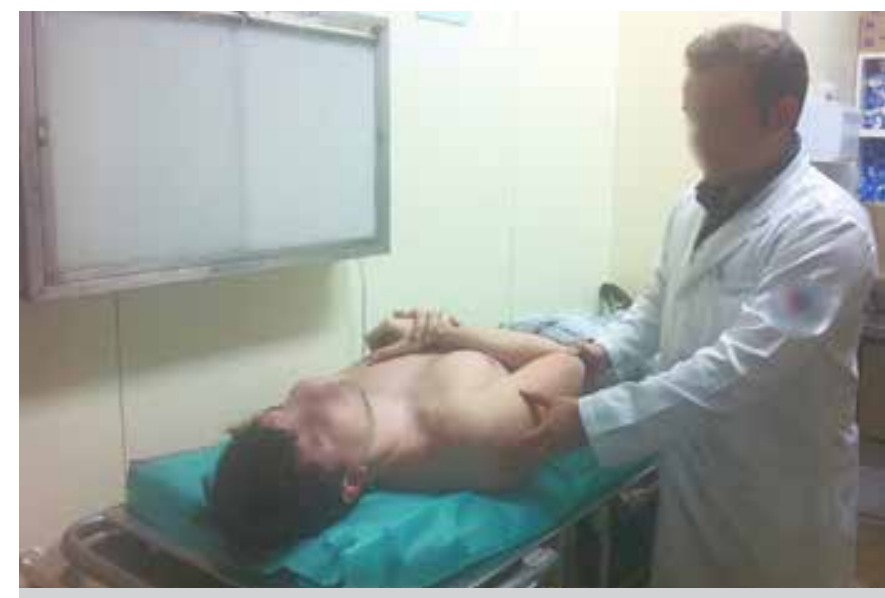

Figure 7. Extension and internal rotation of the shoulder.

\section{DISCUSSION}

Glenohumeral dislocation is a very common diagnosis in orthopedic emergencies and brings to the patient high morbidity and disability. Thus, it is of importance its knowledge and proper management by the orthopedic physician. The classic mechanism of injury is described as indirect trauma on the limb which, in general, is presented in typical attitude, facilitating clinical diagnosis. Confirmation should be obtained by radiographic evaluation with the trauma series - true AP, scapular and axillary profile, as well as the neurovascular status and the presence of previously evaluated concomitant fracture. ${ }^{9}$

Once the preliminary propaedeutic is performed, there should be emergency orthopedic intervention, aiming to relieve pain, bring comfort to the patient and, especially, minimizing damage to the noble and periarticular soft tissue structures. This is where the knowledge of various reduction techniques becomes important in order to obtain better results.

Table 1. Demographic characteristics of patients divided in two groups.

\begin{tabular}{|c|c|c|c|}
\hline \multirow[b]{3}{*}{ Age (years old) } & \multicolumn{2}{|c|}{ Method of reduction } & \multirow{3}{*}{$\begin{array}{l}p \text { value } \\
p=0.787\end{array}$} \\
\hline & Spaso & Kocher & \\
\hline & $30.356 \quad 9.604$ & $30.954 \quad 10.961$ & \\
\hline \multicolumn{4}{|l|}{ Gender } \\
\hline Male & $39(86.7 \%)$ & $36(83.7 \%)$ & $p=0.770$ \\
\hline Female & $6(13.3 \%)$ & $7(16.2 \%)$ & \\
\hline \multicolumn{4}{|l|}{ Side } \\
\hline Right & $28(62.2 \%)$ & $27(62.8 \%)$ & $p=0.956$ \\
\hline Left & $17(37.8 \%)$ & $16(38.2 \%)$ & \\
\hline $\begin{array}{c}\text { Time between dislocation and } \\
\text { care }(\mathrm{h})\end{array}$ & $2.07 \quad 1.3$ & $2.08 \quad 1.64$ & $p=0.398$ \\
\hline Number of previous dislocations & $3.156 \quad 3.49$ & $1.907 \quad 2.234$ & $p=0.283$ \\
\hline
\end{tabular}

Table 2. Reduction results in the two groups.

\begin{tabular}{|c|c|c|c|}
\hline & \multicolumn{2}{|c|}{ Maneuver } & \multirow[t]{2}{*}{$p$ value } \\
\hline & Spaso & Kocher & \\
\hline Efficacy & $40(88.9 \%)$ & $30(69.77 \%)$ & $p=0.0035^{*}$ \\
\hline Average time of reduction (sec) & $46.750(5-180)$ & $213.837(30-180)$ & $p<0.005^{*}$ \\
\hline Pain (VAS) & $3.133(1-5)$ & $3.302(2-5)$ & $p=0.433 \pi$ \\
\hline
\end{tabular}

As mentioned, there is a wide range of maneuvers described, since from the most ancient techniques as Hippocrates, Kocher, ${ }^{1}$ Milch, Stimson, ${ }^{10}$ traction against traction, up to the Spaso technique, published in $1998 .{ }^{4}$

The decision on the maneuver to be carried out depends on the experience of the orthopedist and personal choice, with weak grounding in scientific evidence (evidence level 4 or 5 ), ${ }^{11}$ in view of the lack of comparative studies among the methods. In our work, we opted for the evaluation of a classical method, described by Kocher in 1870, 1,3 known by most orthopedists, cited in most published studies, with efficacy ranging from $71 \%$ to $90 \%, 2,9,12,13$ Our result, however, showed $69.77 \%$ reduction through this technique, with an average time of $213.837 \mathrm{sec}$ and pain level reported by the patients of 3,302 on the visual analogue scale. We compared the Kocher technique to a recent technique, described in 1998 by Miljesic and Kelly, ${ }^{4.14}$ less known by most orthopedic surgeons, but that has shown very good outcomes in the few published works on thetopic. $5,9,12-14$ According to the average described in the literature, we obtained reduction in $88.9 \%$ of cases by Spaso maneuver, taking an average time of 46.75 seconds with pain level of 3.13 on the VAS scale, on average.

The comparative analysis was in favor of Spaso maneuver, with a $p$ value of 0.0035 obtained by chi-square test for effectiveness of the reduction between the techniques. The same outcome was obtained regarding the time required to reduce, by the Mann-Whitney-Wilcoxon method, with a p value less than 0.005 . The only evaluated parameter that did not show significant difference between the groups was VAS.

We can infer from the data analysis, corroborated by hypothesis testing, that the Spaso maneuver was more efficient, fast and reproducible compared to Kocher's, showing also minimal complications and being feasible by a single performer, without previous analgesia.

It is known that the reduction maneuvers vary in nature: traction, lever, scapular manipulation and combined methods. ${ }^{9}$ The Spaso technique fits in traction group; however, it has important biomechanical basis, particularly anatomical.

Adapting what was described by Milch, ${ }^{10,15}$ we can consider that the shoulder has three major vector groups acting on each other, that reflect the local muscle action and act synergistically with a resultant oriented in the medial direction: horizontal (rotator cuff) oblique (teres major, pectoralis major and latissimus dorsi) and vertical (deltoid, biceps and triceps), when in the anatomical position these groups are competing with each other. (Figure 8) The act of bending or elevating the limb promotes an alignment of these vector groups that begin to act in the same direction, minimizing muscular opposition known to play a key role in the failure of any reduction maneuver. (Figure 9) Another relevant aspect is the absence of analgesia, anesthesia or sedation in our protocol. According to some authors, ${ }^{11,14,16}$ analgesic medication may be omitted for the treatment of acute glenohumeral dislocations, especially when employing effective reduction technique, which is fast and presents low morbidity, especially when relying on the patient's cooperation. ${ }^{16}$

It is worth noticing that our sample, although calculated by Lehr formula, ${ }^{6}$ was based on data previously published. Thus, it is not possible to rule out any bias in the number of participants. And we also cannot state that it is a completely safe and 


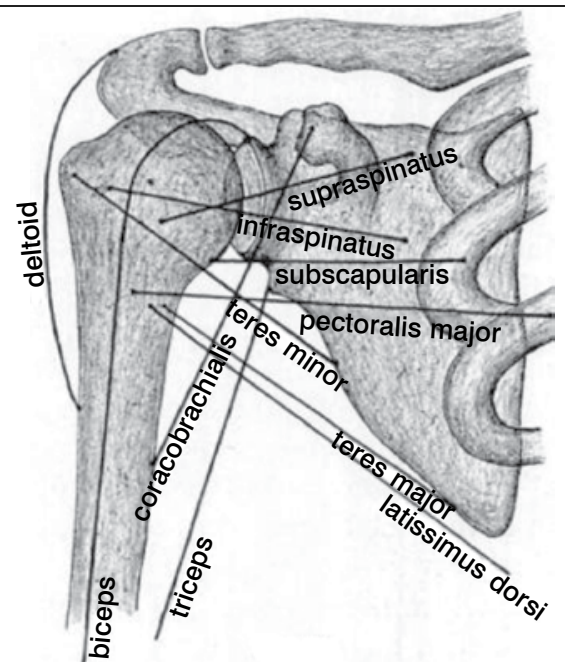

Figure 8. Muscular action on the shoulder in the anatomical position with three vector groups in three different directions: horizontal, vertical and oblique. Source: Milch et al. ${ }^{10}$

effective method, being necessary, therefore, a larger sample group. Furthermore, patients were not previously assessed for ligamentous shoulder instability ${ }^{8}$ - a fact that could interfere with the result of the reduction maneuver, although the groups are demographically comparable, also regarding the number of previous dislocations. Moreover, due to the nature and design of this study, it was not possible masking their participants doctors and patients. We also believe that the results obtained from each group would tend to look alike if anesthesia or sedation would be administered prior to reduction.

Finally, more research is needed on a topic of such importance, especially works that compare the techniques described, since there could be other maneuvers with are equally satisfactory results. ${ }^{11}$

\section{REFERENCES}

1. Kocher T. Eine neue Reductions methode fur Srchulter verrenkung. Berliner Kiln Wchnschr. 1870;7:101-5.

2. Riebel GD, McCabe JB. Anterior shoulder dislocation: a review of reduction techniques. Am J Emerg Med. 1991;9(2):180-8.

3. Uglow MG. Kocher's painless reduction of anterior dislocation of the shoulder: a prospective randomised trial. Injury. 1998;29(2):135-7.

4. Miljesic S, Kelly AM. Reduction of anterior dislocation of the shoulder: the Spaso technique. Emerg Med. 1998;10(3/4):173-5.

5. Fernández-Valencia JA, Cuñe J, Casulleres JM, Carreño A, Prat S. The Spaso technique: a prospective study of 34 dislocations. Am J Emerg Med. 2009;27(4):466-9.

6. Lehr R. Sixteen S-squared over D-squared: a relation for crude sample size estimates. Stat Med. 1992;11(8):1099-102.

7. Petrie A. Statistics in orthopaedic papers. J Bone Joint Surg $\mathrm{Br}$. 2006;88(9):1121-36.

8. Sayegh FE, Kenanidis EI, Papavasiliou KA, Potoupnis ME, Kirkos JM, Kapetanos GA. Reduction of acute anterior dislocations: a prospective randomized study comparing a new technique with the Hippocratic and Kocher methods. J Bone Joint Surg Am. 2009;91(12):2775-82.

9. Filho IAA, Leitão ICS, Castro L, Neto PJP. Luxação glenoumeral anterior aguda:

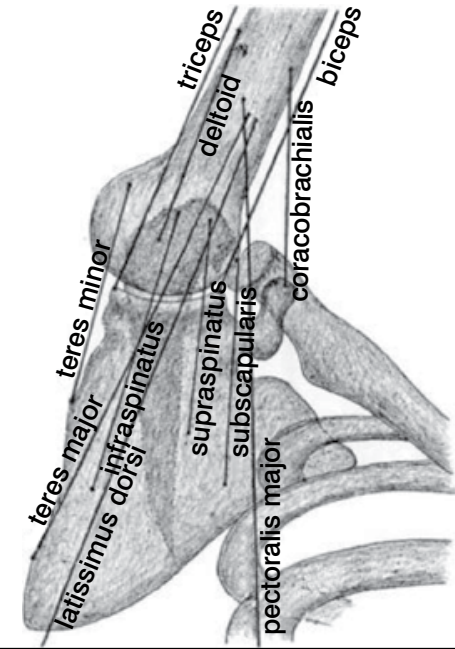

Figure 9. Muscular action on the elevated shoulder. Notice the alignment of the vector groups that start acting in the same direction Source: Milch et al. ${ }^{10}$

\section{CONCLUSION}

We conclude that the Spaso maneuver is efficient, fast and easily reproducible, promoting the reduction of glenohumeral dislocations in a secure manner. It can also be carried out by one single person after relatively little training or experience and without analgesic drugs, muscle relaxants, anesthetics or sedatives. It also presents better results when compared to Kocher's maneuver regarding effectiveness and speed in reducing glenohumeral dislocations. There were no differences in pain levels during reduction for both techniques.

More comparative studies are needed to compare the various existing techniques in order to develop protocols aimed at improving the quality of care provided. estudo comparativo entre métodos de redução incruenta. Rev Bras Ortop. 2006;41(11/12):455-60.

10. Milch $\mathrm{H}$. The treatment of recent dislocations and fracture-dislocations of the shoulder. J Bone Joint Surg Am. 1949;31(1):173-80.

11. Kuhn JE. Treating the initial anterior shoulder dislocation--an evidence-based medicine approach. Sports Med Arthrosc. 2006;14(4):192-8.

12. Yuen MC, Tung WK. The use of the Spaso technique in a patient with bilateral dislocations of shoulder. Am J Emerg Med. 2001;19(1):64-6.

13. Yuen MC, Yap PG, Chan YT, Tung WK. An easy method to reduce anterior shoulder dislocation: the Spaso technique. Emerg Med J. 2001;18(5):370-2.

14. Ugras AA, Mahirogullari M, Kural C, Erturk AH, Cakmak S. Reduction of anterior shoulder dislocations by Spaso technique: clinical results. J Emerg Med. 2008;34(4):383-7

15. Milch H. Treatment of dislocation of the shoulder. Surgery. 1938;3:73240. Wainsztein A, Tamaoki MJS, Archetti Netto N, Belotti JC, Matsmoto MH, Faloppa F. Estudo transversal sobre os diferentes métodos de tratamento das luxações traumáticas glenoumerais. Rev Bras Ortop. 2009;44(5):391-6.

16. O'Connor DR, Schwarze D, Fragomen AT, Perdomo M. Painless reduction of acute anterior shoulder dislocations without anesthesia. Orthopedics. 2006;29(6):528-32. 